\title{
Equivalence classes of permutations modulo excedances
}

\author{
Jean-Luc Baril, Toufik Mansour, and Armen Petrossian
}

\begin{abstract}
We introduce a new equivalence relation on permutations where two permutations are equivalent if and only if they coincide on their excedance sets. This paper studies equivalence classes for several subsets of permutations. Enumerating results are presented for permutations, cycles and permutations avoiding one or two patterns of length three. Also, an open question is proposed.
\end{abstract}

Keywords And Phrases: Permutations, equivalence class, excedance, pattern, Bell, Motzkin, Catalan, Fibonacci numbers.

\section{Introduction and notations}

Let $S_{n}$ be the set of permutations of length $n$, i.e., all one-to-one correspondences from $[n]=\{1,2, \ldots, n\}$ into itself. The one-line notation of a permutation $\pi \in S_{n}$ is $\pi_{1} \pi_{2} \cdots \pi_{n}$ where $\pi_{i}=\pi(i)$ for $i \in[n]$. A cycle in $S_{n}$ is a $n$-length permutation $\pi$ such that there exist some indices $i_{1}, i_{2}, \ldots, i_{n}$ with $\pi\left(i_{1}\right)=i_{2}, \pi\left(i_{2}\right)=i_{3}, \ldots, \pi\left(i_{n-1}\right)=i_{n}$ and $\pi\left(i_{n}\right)=i_{1}$. According to the context, a cycle will be also denoted by its cyclic notation $\pi=\left\langle i_{1}, i_{2}, \ldots, i_{n}\right\rangle$. Let $C_{n} \subset S_{n}$ be the set of all cycles of length $n$.

Let $\pi$ be a permutation in $S_{n}$. An excedance of $\pi$ is a position $i, 1 \leq i \leq n$, such that $\pi(i)>i$. The set of excedances of $\pi$ will be denoted $E(\pi)$. For instance, if $\pi=4163572$ then $E(\pi)=\{1,3,6\}$. The graphical representation of $\pi$ is the set of points in the plane at coordinates $\left(i, \pi_{i}\right)$ for $i \in[n]$. We define its associated rook placement $R(\pi)$ on the triangular board consisting of the cells located atop the diagonal $y=x$ in the graphical representation of the permutation. For short, $R(\pi)$ will be called excedance board of $\pi$. Obviously, a point $\left(i, \pi_{i}\right)$ appears in the excedance board of $\pi$ if and only if $i$ is an excedance of $\pi$. See Figure 1 for the graphical representation of a permutation with its associated excedance board.

We consider the equivalence relation $\sim$ on $S_{n}$ in which two permutations $\pi$ and $\sigma$ are considered to be equivalent if they coincide on their excedance sets, i.e., $E(\pi)=E(\sigma)$ and $\pi(i)=\sigma(i)$ for $i \in E(\pi)$, or again $\pi$ and $\sigma$ have the same excedance board. Let $\mathrm{Eq}\left(\pi, S_{n}\right)$ denote the set of permuta- 

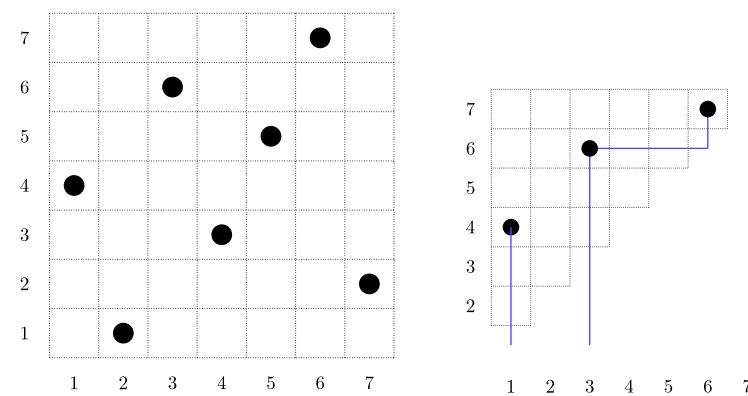

$14 / 2 / 367 / 5$

Figure 1: Graphical representation of $\sigma=4163572$ with its excedance board and its associated partition.

tions being equivalent to $\pi$ in $S_{n}$. For instance, we have $\operatorname{Eq}\left(43215, S_{5}\right)=$ $\{43215,43125\}$. The set (resp. the cardinality of the set) of distinct equivalence classes in $S_{n}$ is denoted $S_{n}^{\sim}\left(\operatorname{resp} . \# S_{n}^{\sim}\right)$. All these definitions will remain available by replacing the set $S_{n}$ with a subset of $S_{n}$.

The theory of permutation statistics has a long history and has grown at a rapid place in the last few decades. Two classical statistics have been received lot of attention, namely number of descents and number of excedances in a permutation (see [5] and references therein). Recently, Elizalde [6] studied the number of permutations that avoid a fixed set $T$ of patterns according to the number of fixed points and excedances. In this paper, we study the number of distinct equivalence classes in either $S_{n}^{\sim}$ or $S_{n}^{\sim}(T)$ with a fixed set $T$ of patterns.

A permutation $\pi \in S_{n}$ avoids the pattern $\tau \in S_{k}$ if and only if there does not exist a sequence of indices $1 \leq i_{1}<i_{2}<\cdots<i_{k} \leq n$ such that $\pi\left(i_{1}\right) \pi\left(i_{2}\right) \ldots \pi\left(i_{k}\right)$ is order-isomorphic to $\tau$ (see $[13,14]$ ). We denote by $S_{n}(\tau)$ the set of permutations of $S_{n}$ avoiding the pattern $\tau$. For example, if $\tau=123$ then $52143 \in S_{5}(\tau)$ while $21534 \notin S_{5}(\tau)$. Many classical sequences in combinatorics appear as the cardinality of pattern-avoiding permutation classes. A large number of these results were firstly obtained by West and Knuth [9, 12, 13, 14, 15, 16] (see books of Kitaev [8] and Mansour [11]).

In Section 2, we prove that equivalence classes for $S_{n}$ are enumerated by the $n$th Bell number. We also prove that the number of classes in $C_{n}$ is given by the sequence of the number of irreducible set partitions of $[n]$ (see A074664 in the on-line Encyclopedia of Integer Sequences [17]). In Section 3, we give enumerating results for permutations avoiding one pattern of length three (see Table 1). When the pattern is 132, the number of classes is given by the Motzkin numbers which provides a kind of discrete continu- 
Table 1: Number of equivalence classes for permutations avoiding at most one pattern of $\mathcal{S}_{3}$, where $B_{n}$ (resp. $M_{n}$ ) are the Bell numbers (resp. Motzkin numbers) defined by the generating function $\sum_{k \geq 0} \frac{x^{k}}{\prod_{j=1}^{k}(1-j x)}$ (resp. $\left.\frac{1-x-\sqrt{1-2 x-3 x^{2}}}{2 x^{2}}\right)$

\begin{tabular}{|c|c|c|c|}
\hline Pattern & Sequence & Sloane & $a_{n}, n \geq 1$ \\
\hline\{\} & $B_{n}$ & $A 000110$ & $1,2,5,15,52,203,877,4140,21147$ \\
\hline$\{132\},\{213\}$ & $M_{n}$ & $A 001006$ & $1,2,4,9,21,51,127,323,835$ \\
\hline$\{123\}$ & Open question & New & $1,2,4,8,19,41,98,221,526$ \\
\hline$\{312\},\{321\}$ & $\frac{1}{n+1}\left(\begin{array}{c}2 n \\
n\end{array}\right)$ & $A 000108$ & $1,2,5,14,42,132,429,1430,4862$ \\
\hline$\{231\}$ & & $N e w$ & $1,2,4,9,21,50,121,296,729$ \\
\hline
\end{tabular}

ity between Catalan and Motzkin numbers. The case where permutations avoid the pattern 123 remains an open question. In Section 4, we investigate permutations avoiding two patterns of length three (see Table 3 ).

\section{Equivalence classes for $S_{n}$ and $C_{n}$}

A partition of $[n]$ is any collection of non-empty pairwise disjoint subsets, called blocks, whose union is $[n]$. Let $\mathcal{P}_{n}$ be the set of partitions of $[n]$. A partition $\Pi$ is said to be in standard form if it is written $\Pi=B_{1} / B_{2} /$ $\cdots$, where the blocks $B_{i}$ are arranged so that their smallest elements are in increasing order. For convenience, we assume also that elements in a same block are arranged in increasing order. An atomic partition is a partition that does not have a proper subset of blocks with a union equal to a subset $[j]$ with $1 \leq j \leq n-1$.

From a permutation $\pi \in S_{n}$, we associate the partition $\Pi \in \mathcal{P}_{n}$ defined as follows. Two elements $x<y$ belong to the same block in $\Pi$ if and only if there exist $k \geq 1$ and $i_{1}=x<i_{2}<\cdots<i_{k}=y$ such that $\pi_{i_{j}}=i_{j+1}$ for $1 \leq j \leq k-1$. For instance, the partition associated to $\pi=4163572$ is $\Pi=14 / 2 / 367 / 5$ (see Figure 1). Indeed, if $i_{1}=1$ then we have $i_{1}=$ $1<i_{2}=\pi_{i_{1}}=4$ and $\pi_{i_{2}}=3<4$ which induces that 14 is a block; if $i_{1}=2$ then we have $\pi_{i_{1}}=1<2$, so 2 is a block; if $i_{1}=3$ then we have $i_{1}=3<i_{2}=\pi_{i_{1}}=6<i_{3}=\pi_{i_{2}}=7$ and $\pi_{i_{3}}=2<7$, so 367 forms a block, and so on. Notice that this definition appears as a counterpart for permutations of the partition associated to a rook placement on a Ferrers board (see Stanley [18], p. 75, or Mansour [10], p. 99).

\subsection{For $S_{n}-$ Bell numbers}

Theorem 2.1. The sets $S_{n}^{\sim}, n \geq 1$, are enumerated by the Bell numbers. 
Proof. Two permutations lie in a same equivalence class $\operatorname{Eq}\left(\pi, S_{n}\right)$ if and only if they have the same excedance board $R(\pi)$. Then they have the same associated partition. Moreover, the associated partitions of two nonequivalent permutations are different.

Conversely, let $\Pi=B_{1} / B_{2} / \cdots / B_{k}$ be a partition of $[n]$ in the standard form and such that each block $B_{i}, 1 \leq i \leq k$ is arranged in increasing order. We define the permutation $\pi \in S_{n}$ such that $\pi_{i}=j$ if $j$ is just after $i$ into a same block ( $j$ is the smallest element greater than $i$ in the block) and the image by $\pi$ of the largest element of a block is the smallest element of this block. For instance, if $\Pi=14 / 2 / 367 / 5$ we obtain the permutation $\pi=4261573$. By construction, this process defines a permutation in $S_{n}$ such that $\Pi$ is its associated partition. So, there is a bijection between $S_{n}^{\sim}$ and the set $\mathcal{P}_{n}$ of partitions of $[n]$. Therefore, $S_{n}^{\sim}$ is enumerated by the $n$-th Bell number.

\subsection{For $C_{n}-\mathrm{A} 074664$}

Lemma 1. The associated partition of a cyclic permutation $\pi \in C_{n}$ is atomic.

Proof. For $\pi \in C_{n}$, let $\Pi=B_{1} / B_{2} / \cdots / B_{k}$ be its associated partition in standard form. For a contradiction, we assume that $\Pi$ is not atomic, i.e., there exists a proper subset of blocks $B_{1}, B_{2}, \ldots, B_{\ell}, \ell<k$ with a union equal to a subset $[j]$ for some $j \in[n-1]$. Then for any $i \in[j]$, we have $\pi_{i} \in[j]$. Indeed, if there was $i \leq j$ such that $\pi_{i}>j$, then $i$ would be an excedance and $\pi_{i}$ would be in a same block. This case does not occur since the hypothesis induces that the block containing $i$ and $\pi_{i}$ is included in $[j]$. Therefore, the image by $\pi$ of the interval $[j], j<n$, still remains $[j]$, which contradicts the fact that $\pi$ is a cycle in $C_{n}$.

Theorem 2.2. The sets $C_{n}^{\sim}, n \geq 1$, are enumerated by the sequence A074664 in [17].

Proof. The previous lemma proves that the associated partition of a cycle in $C_{n}$ is necessarily atomic. Moreover, the associated partitions of two non-equivalent cycles are different. Now, we will prove that for any atomic partition $\Pi \in \mathcal{P}_{n}$, there exists a cycle $\pi \in S_{n}$ having $\Pi$ as associated partition (see the definition on the previous page). Let $\Pi=B_{1} / B_{2} / \cdots / B_{k}$ be an atomic partition (not necessarily in standard form). Let us assume that $1 \in B_{1}$ and put $i_{1}=1$. For $1 \leq i \leq k$, we denote by $M\left(B_{i}\right)$ (resp. $m\left(B_{i}\right)$ ) the maximum (resp. minimum) element in the block $B_{i}$. In the case where $M\left(B_{1}\right) \neq n$, the atomicity of $\Pi$ induces that there is a block $B_{i_{2}}$ such that $1<m\left(B_{i_{2}}\right)<M\left(B_{i_{1}}\right)<M\left(B_{i_{2}}\right)$. We choose the block $B_{i_{2}}$ having 
its smallest element the lowest possible. Iterating this process by replacing $B_{i_{1}}$ with $B_{i_{2}}$, we exhibit a sequence of blocks $B_{i_{1}}, B_{i_{2}}, \ldots, B_{i_{r}}$ such that $m\left(B_{i_{j}}\right)<m\left(B_{i_{j+1}}\right)<M\left(B_{i_{j}}\right)<M\left(B_{i_{j+1}}\right)$ for $1 \leq j \leq r-1$ and such that $B_{i_{r}}$ contains the value $n$. Let $\pi$ be the cycle in $C_{n}$ defined as follows: $\pi=\left\langle B_{i_{1}} B_{i_{2}} \cdots B_{i_{r}} A_{1} A_{2} \cdots A_{s}\right\rangle$ where each block $B_{i_{j}}, 1 \leq j \leq r$ has its elements in increasing order, and the blocks $A_{i}, 1 \leq i \leq s$, are the remaining blocks of the partition $\Pi$ ordered by their decreasing minima and each of them ordered in increasing order. By construction $\pi$ is a cycle in $C_{n}$, and it is straightforward to see that $\Pi$ is its associated partition. Using Lemma 1, we have exhibited a constructive bijection between $C_{n}^{\sim}, n \geq 1$, and the subset of atomic partitions in $\mathcal{P}_{n}$ which is enumerated by the sequence A074664 in [17] (see Bergeron and Zabrocki [4]).

A permutation $\pi=\pi_{1} \pi_{2} \cdots \pi_{n} \in S_{n}$ is called indecomposable if there does not exist $i<n$ such that $\pi_{1} \pi_{2} \cdots \pi_{i}$ is a permutation of $[i]$. Let $\operatorname{In} d_{n}$, $n \geq 1$, be the sets of indecomposable permutations of length $n$. Lemma 1 remains valid if we replace cyclic permutation with indecomposable permutation. Moreover, since a cyclic permutation is necessarily indecomposable, the proof of the following theorem is obtained, mutatis mutandis, from the proof of Theorem 2.2.

Theorem 2.3. The sets $\operatorname{Ind}_{n}^{\sim}, n \geq 1$, are enumerated by the sequence A074664 in [17].

\section{Equivalence classes for $S_{n}(\alpha)$ with $\alpha \in S_{3}$}

In this section, we study enumerating results about the equivalence classes of sets of permutations avoiding one pattern of length three.

For the pattern 321, the excedances of $\pi \in S_{n}(321)$ determine $\pi$ completely which means that every permutation $\pi \in S_{n}(321)$ is the unique element in its equivalence class. Therefore, the cardinality of the set $S_{n}^{\sim}(321)$ is given the $n$-th Catalan number (see sequence A000108 in [17]).

For the pattern 312, we use the Simion-Schmidt bijection modulo symmetry from $S_{n}(321)$ to $S_{n}(312)$ (see [13]). Since this bijection preserves the position of points $\left(i, \pi_{i}\right)$ whenever $\pi_{i}>i$, we directly conclude that the cardinality of the set $S_{n}^{\sim}(312)$ is given by the cardinality of $S_{n}(312)$, that also is the $n$-th Catalan number. We have not succeeded in finding the cardinality of $S_{n}^{\sim}(123)$ for any $n \geq 1$ (first values are $1,2,4,8,19,41,98,221,526$ ). So, we leave this problem open.

In order to study other patterns, we define some other equivalence relations between two permutations by extending the definition of an excedance. Let $\pi$ be a permutation in $S_{n}$. A $k$-excedance of $\pi, 1-n \leq k \leq n$, is a po- 
sition $i, 1 \leq i \leq n$, such that $\pi(i)>i-k$. The set of $k$-excedances of $\pi$ will be denoted $E^{k}(\pi)$. For instance, if $\pi=312$ then $E^{-2}(\pi)=\emptyset, E^{-1}(\pi)=$ $E^{0}(\pi)=E^{1}(\pi)=\{1\}$ and $E^{2}(\pi)=[3]$. Obviously, for any $\pi \in S_{n}$, we have $E^{0}(\pi)=E(\pi), E^{1-n}(\pi)=\emptyset$ and $E^{n}(\pi)=[n]$. For any $k, 1-n \leq k \leq n$, we define the $k$-equivalence relation $\sim^{k}$ on $S_{n}$ in which two permutations $\pi$ and $\sigma$ are equivalent if and only if they coincide on their $k$-excedance sets, i.e., $\pi(i)=\sigma(i)$ for $i \in E^{k}(\pi)=E^{k}(\sigma)$. Clearly, when $k=0$ we retrieve the equivalence relation defined in the introduction. Let $\mathrm{Eq}^{k}\left(\pi, S_{n}\right)$ denote the set of permutations in $S_{n} k$-equivalent to $\pi$; we call it the $k$-class of $\pi$. For instance, we have $\operatorname{Eq}^{0}\left(43215, S_{5}\right)=\operatorname{Eq}\left(43215, S_{5}\right)=\{43215,43125\}$, $\operatorname{Eq}^{2}\left(43215, S_{5}\right)=\{43215\}$ and $\operatorname{Eq}^{-4}\left(43215, S_{5}\right)=S_{5}$. The set of distinct $k$-classes in $S_{n}$ is denoted $S_{n}^{\sim^{k}}$.

\subsection{The sets $S_{n}^{\sim}(132)$ and $S_{n}^{\sim}(213)-$ Motzkin}

We focus our study on the pattern 132 and the symmetry reverse $\rightarrow$ complement $\rightarrow$ inverse will provide the result for 213 .

Theorem 3.1. The sets $S_{n}^{\sim}(132), n \geq 1$, are enumerated by the Motzkin numbers.

Proof. For $k \geq 0$, let $M_{n}^{k}$ be the number of $k$-classes in $S_{n}(132)$. Let $\pi$ be a permutation in $S_{n}(132)$. Since $\pi$ avoids the pattern $132, \pi$ can be written $\pi=\sigma \gamma i, 1 \leq i \leq n$, where $\gamma i$ is a subsequence of $[i]$ avoiding the pattern 132 and $\sigma$ is obtained from a permutation $\sigma^{\prime} \in S_{n-i}(132)$ by adding $i$ on all its entries, i.e., $\sigma(j)=i+\sigma^{\prime}(j)$ for $1 \leq j \leq n-1-i$.

We distinguish two cases: (1) $i \geq n-k$, and (2) $i \leq n-k-1$.

Case 1 . In this case, $n$ is either a $k$-excedance (i.e., $\pi_{n}=i \geq n-k+1$ ) or $\pi_{n}=i=n-k$. The decomposition of $\pi=\sigma \gamma i$ is illustrated by the left part of Figure 2. The blue (resp. green) square corresponds to $\sigma$ (resp. $\gamma$ ), and the filled gray area does not contain any point $\left(j, \pi_{j}\right)$.

It is straightforward and crucial to see that for a given $i, i \geq n-k$, the above decomposition of $\pi$ induces that:

an integer $j>n-i$ is a $k$-excedance of $\pi$ if and only if $j-n+i$ is $a(k+i-n)$-excedance of $\gamma i$ where $\gamma i$ is considered as a permutation in $S_{i}(132)$.

Using the decomposition of $\pi=\sigma \gamma i$, the number of $k$-classes having a representative ending by $i \geq n-k$ is equal to the product between the number of $(k+i-n)$-classes of $S_{i-1}(132)$ and the number of subsequences $\sigma$ of length $n-i$ avoiding 132 , that is $M_{i-1}^{k+i-n} \cdot c_{n-i}$ where $c_{n-i}=\frac{1}{n-i+1}\left(\begin{array}{c}2(n-i) \\ n-i\end{array}\right)$ is the $(n-i)$ th Catalan number. Varying $i$ from $n-k$ to $n$, the number of 

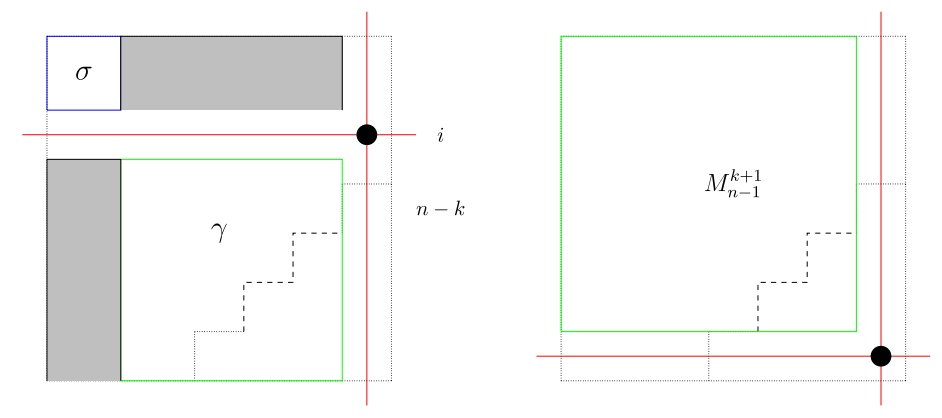

Figure 2: Illustration of the two cases studied in the proof of Theorem 4 . (Color figure online)

$k$-classes having a representative with a last element greater or equal than $n-k$ is exactly $M_{n-1}^{k} c_{0}+M_{n-2}^{k-1} c_{1}+\ldots+M_{n-k-1}^{0} c_{k}$.

Now, let us examine the second case.

Case 2. We assume $i \leq n-k-1$ and we will prove that each $k$-class contains a permutation having its last element equal to 1 . Let $\pi$ be a permutation such that $\pi_{n}=i \leq n-k-1$ and let us assume that $\pi_{n}=i \neq 1$. Using the decomposition $\pi=\sigma \gamma i$, the value 1 appears in $\gamma$, i.e., $\pi_{j}=1$ implies $j \geq n-i+1$. Since $\pi$ avoids 132 , all values after $\pi_{j}=1$ appear in increasing order in the one-line representation of $\pi$ and are less or equal than $i$. This implies that for $\ell \geq j, \pi_{\ell}$ are less or equal than $i-(n-\ell)$.

Now, let us define the permutation $\pi^{\prime} \in S_{n}(132)$ such that $\pi_{n}^{\prime}=1$, $\pi_{\ell}^{\prime}=\pi_{\ell}$ if $\ell \in[j-1]$, and $\pi_{\ell}^{\prime}=\pi_{\ell+1}$ for $\ell \in[j, n-1]$. By construction, $\pi^{\prime} \in S_{n}(132)$ and $\pi$ belongs to the same $k$-class. Thus, the number of $k$ classes (in this case) is equal to the number of $(k+1)$-classes in $S_{n-1}(132)$ (we omit the last value 1 of $\pi^{\prime}$ ), which is given by $M_{n-1}^{k+1}$ (see the right part of Figure 2).

In accordance with the two previous cases, we obtain the following recursive formula for $k \geq 0, n \geq 1$ and $n>k$ :

$$
M_{n}^{k}=M_{n-1}^{k+1}+M_{n-1}^{k} c_{0}+M_{n-2}^{k-1} c_{1}+\ldots+M_{n-k-1}^{0} c_{k}
$$

anchored with $M_{n}^{n}=c_{n}$ for $n \geq 0$, where $c_{n}$ is the $n$th Catalan number (see A000108 in [17]).

Setting $D^{n}(x)=\sum_{k \geq 0} M_{k+n}^{k} x^{k}$ for $n \geq 1$, the bivariate generating function $F(x, u)$ where the coefficient of $x^{k} u^{n}$ is the number of $k$-classes in $S_{n+k}(132)$ satisfies $F(x, u)=\sum_{n \geq 1} D^{n}(x) u^{n}$. A simple calculation from 
Table 2: Number $M_{n}^{k}$ of $k$-classes in $S_{n}(132)$ for some $n$ and $k, n>k \geq 0$

\begin{tabular}{c|ccccccc}
$k \backslash n$ & 1 & 2 & 3 & 4 & 5 & 6 & 7 \\
\hline 0 & 1 & 2 & 4 & 9 & 21 & 51 & 127 \\
1 & & 2 & 5 & 12 & 30 & 76 & 196 \\
2 & & & 5 & 14 & 37 & 99 & 265 \\
3 & & & & 14 & 42 & 118 & 331 \\
4 & & & & & 42 & 132 & 387 \\
5 & & & & & & 132 & 429
\end{tabular}

the previous recurrence relation provides the functional equation for $n \geq 3$ :

$$
D^{n}(x)=C(x) D^{n-1}(x)+\frac{D^{n-2}(x)-D^{n-2}(0)}{x}
$$

with $D^{1}(x)=\frac{C(x)-1}{x}$ and $D^{2}(x)=\frac{D^{1}(x)-1}{x}$ where $C(x)=\frac{1-\sqrt{1-4 x}}{2 x}$ is the generating function for the Catalan numbers.

With this last relation, we obtain:

$$
F(x, u)\left(x-C(x) x u-u^{2}\right)=D^{1}(x) x u+D^{2}(x) x u^{2}-C(x) x D^{1}(x) u^{2}-u^{2} F(0, u) .
$$

Using the kernel method (see $[1,9]$ ), we compute

$$
x=\frac{u}{2}\left(1+u-2 u^{2}-\sqrt{1-2 u-3 u^{2}}\right) .
$$

This induces $F(0, u)=\frac{1-u-\sqrt{1-2 u-3 u^{2}}}{2 u^{2}}$ which is the generating function for the Motzkin numbers. Therefore, $M_{n}^{0}$ is the $n$th term of the Motzkin sequence.

Finally, a simple calculation provides

$$
F(x, u)=\frac{u-u \sqrt{1-4 x}-u x+u^{2}-u^{2} \sqrt{1-4 x}-x+x \sqrt{1-2 u-3 u^{2}}}{x\left(2 x-u+u \sqrt{1-4 x}-2 u^{2}\right)} .
$$

The bivariate generating function obtained in the proof of Theorem 3.1 provides a kind of discrete continuity between the well-known Catalan and Motzkin sequences (see Table 2). See [3] for another discrete continuity between these sequences and [2] for a discrete continuity between Fibonacci and Catalan sequences. Using Maple, the first terms of the Taylor expansion of $F(x, u)$ are $u+2 u^{2}+4 u^{3}+9 u^{4}+21 u^{5}+51 u^{6}+127 u^{7}+2 x u+5 x u^{2}+$ $12 x u^{3}+30 x u^{4}+76 x u^{5}+196 x u^{6}+5 x^{2} u+14 x^{2} u^{2}+37 x^{2} u^{3}+\ldots$. 


\subsection{The set $S_{n}^{\sim}(231)$}

Theorem 3.2. The generating function for the number of classes in $S_{n}(231)$, $n \geq 1$, is given by the continued fraction:

$$
\frac{1}{1+\left[a_{1}\right]-\frac{x}{1+\left[a_{2}\right]-\frac{x}{1+\left[a_{3}\right]-\frac{x}{1+\left[a_{4}\right]+\cdots}}}}
$$

where $\left[a_{\ell}\right]=\frac{x^{2}\left(1-x^{\ell-1}\right)}{1-x}$ for $\ell \geq 1$.

Proof. For $k \leq 0$, let $M_{n}^{k}$ be the number of $k$-classes in $S_{n}(231)$. Recall that two permutations $\sigma$ and $\pi$ lie in a same $k$-class if and only if they coincide on positions $i$ satisfying $\pi_{i}>i-k$ or $\sigma_{i}>i-k$. Let $\pi$ be a permutation in $S_{n}(231)$ and denote by $i, 1 \leq i \leq n$, the index such that $\pi_{i}=n$. Since $\pi$ avoids the pattern 231, $\pi$ can be written $\pi=\sigma n \gamma$, where $\sigma$ is a permutation of $[i-1]$ avoiding the pattern 231 and $\gamma$ is obtained from a permutation $\gamma^{\prime} \in S_{n-i}(231)$ by adding $i-1$ on all its entries, i.e., $\gamma(j)=i-1+\gamma^{\prime}(j)$ for $i+1 \leq j \leq n$.

We distinguish two cases: (1) $i \leq n-1+k$, and (2) $i>n-1+k$.

Case 1. In this case, $i$ is a $k$-excedance since $\pi_{i}=n>i-k+1>i-k$. Notice that for a given $i, i \leq n-1+k$, the above decomposition of $\pi$ induces that:

an integer $j \neq i$ is a $k$-excedance of $\pi$ if and only either $j$ is a $k$-excedance of $\sigma$, or $j-i+1$ is a $(k-1)$-excedance of $\gamma^{\prime}$ considered as a permutation in $S_{n-i}(231)$.

Thus, the number of $k$-classes having a representative with $n$ in position $i$ is equal to the product $M_{i-1}^{k} \cdot M_{n-i}^{k-1}$. Varying $i$ from 1 to $n-1+k$, the number of $k$-classes in this case is exactly $\sum_{i=0}^{n-2+k} M_{n-1-i}^{k-1} M_{i}^{k}$.

Now, let us examine the second case.

Case 2. We have $i>n-1+k$ and $i$ is not a $k$-excedance. We will prove that the $k$-class of $\pi$ contains a permutation having its last element equal to $n$.

Let us consider the permutation $\pi^{\prime}$ defined by $\pi^{\prime}=\sigma \gamma n$. It is straightforward to check that $\pi^{\prime}$ avoids 231 and that $\pi^{\prime}$ belongs to the same $k$-class of $\pi$. Thus, the number of $k$-classes (in this case) is equal to the number of 
$k$-classes having a representative ending with $n$, which is given by $M_{n-1}^{k}$. In accordance with the two previous cases, we obtain the following recursive formula for $k \leq 0$ and $n \geq 1$ :

$$
\begin{cases}M_{n}^{k}=M_{n-1}^{k}+\sum_{\ell=0}^{n-2+k} M_{\ell}^{k} M_{n-1-\ell}^{k-1} & \text { for } n-1>-k \\ M_{n}^{k}=1 & \text { for } n-1 \leq-k .\end{cases}
$$

Let $D^{k}(x)=\sum_{n \geq 0} M_{n}^{k} x^{n}$ be the generating function for the number of $k$-classes in $S_{n}(231)$. Some elementary calculations from the previous recurrence relation provide the following functional equation for $k \leq 0$ :

$$
D^{k}(x)\left(1+x\left(\frac{1-x^{-k}}{1-x}-1\right)-x D^{k-1}(x)\right)=1 .
$$

Finally, we obtain $D^{k}(x)$ expressed as a continued fraction

$$
\frac{1}{1+\left[a_{-k+1}\right]-\frac{x}{1+\left[a_{-k+2}\right]-\frac{x}{1+\left[a_{-k+3}\right]-\frac{x}{1+\left[a_{-k+4}\right]+\cdots}}}}
$$

where $\left[a_{\ell}\right]=\frac{x^{2}\left(1-x^{\ell-1}\right)}{1-x}$ for $\ell \geq 1$. When $k=0$, we obtain the expected result.

By the equation of $D^{k}(x)$ (see line 4 ), we see that

$$
\lim _{k \rightarrow-\infty} D^{k}(x)\left(1-x+x \frac{1-x^{-k}}{1-x}-x D^{k-1}(x)\right)=1 .
$$

Let $D(x)=\lim _{k \rightarrow-\infty} D^{k}(x)$ and assume that $|x|<1$. Then we have

$$
D(x)\left(1-x+\frac{x}{1-x}-x D(x)\right)=1
$$

which implies that either $D(x)=1 /(1-x)$ or $D(x)=(1-x) / x$. Since $D^{k}(0)=1$ for all $k<0$, we have that $D(0)=1$. Hence, $D(x)=$ $\lim _{k \rightarrow-\infty} D^{k}(x)=\frac{1}{1-x}$

Finally, the first terms of the Taylor expansion of the continued fraction $D^{0}(x)$ can be obtained by replacing the rest of the continued fraction by its 
limit $\frac{1}{1-x}$. More precisely, if we need the first five terms then we expand the rational fraction

$$
\frac{1}{1+\left[a_{1}\right]-\frac{x}{1+\left[a_{2}\right]-\frac{x}{1+\left[a_{3}\right]-\frac{x}{1+\left[a_{4}\right]+\frac{x}{1-x}}}}}
$$

which provides $1+x+2 x^{2}+4 x^{3}+9 x^{4}+\mathcal{O}\left(x^{5}\right)$.

\section{Equivalence classes for $S_{n}(\alpha, \beta)$ with $\alpha$ and $\beta$ in $S_{3}$}

In this part, we give enumerating results for classes of permutations avoiding two patterns of length three. Below, we present proofs for the most interesting cases which appear in the last three rows of Table 3, i.e., for the cases where the equivalence classes are not all reduced to a single permutation. All other cases can be easily proved using similar arguments or by applying classical symmetries on permutations (inverse, reverse, and complement).

\subsection{The set $S_{n}^{\sim}(123,231)-A 096777$}

Theorem 4.1. The number of classes in $S_{n}^{\sim}(123,231), n \geq 1$, is given by

$$
1+\frac{1}{2} \cdot\left\lceil\frac{n-2}{3}\right\rceil \cdot\left(1-2 n+3\left\lceil\frac{n-2}{3}\right\rceil\right)+\left\lfloor\frac{n}{2}\right\rfloor \cdot\left(2 n-1-2\left\lfloor\frac{n}{2}\right\rfloor\right)
$$

(see sequence A096r7y in [17]).

Proof. Let $\pi$ be a permutation in $S_{n}(123,231)$. For $k \in[n+1]$, we define the subsequence $\sigma(k)=n(n-1) \ldots(n-k+1)$ if $k \leq n$, and $\sigma(k)$ is empty if $k=n+1$. Thus, a permutation $\pi \in S_{n}(123,231)$ has a unique decomposition of the form $\pi=\sigma(k) \gamma(\ell) \delta$ where $\delta=(n-k) \cdots(\ell+1)$ and $\gamma(\ell)=\ell(\ell-1) \cdots 21$ with $k \in[n+1]$ and $\ell \in[n-k-1]$ (see Figure 3 for an illustration of this decomposition).

Let $a_{n}$ (respectively $b_{n}^{k}$ ) be the number of classes in $S_{n}(123,231$ ) (respectively starting with $\sigma(k)$ for $\left.k \leq\left\lfloor\frac{n}{2}\right\rfloor\right)$. The above decomposition induces that

$$
a_{n}=1+\sum_{k=1}^{\left\lfloor\frac{n}{2}\right\rfloor-1} b_{n}^{k}+(n-1)
$$




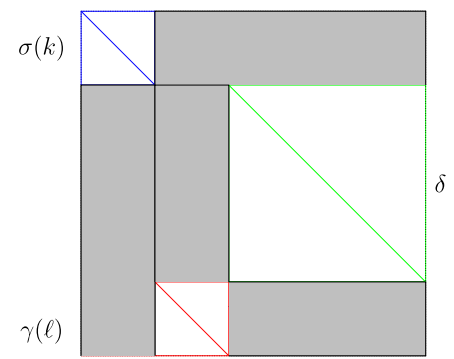

Figure 3: Illustration of the decomposition of a permutation in $S_{n}(123,231)$. (Color figure online)

for $n \geq 1$. Indeed, if $k \geq\left\lfloor\frac{n}{2}\right\rfloor$ then $\sigma(k)$ fills the semi-diagonal $y=n-x+1$ in the excedance board, and $\pi$ and $n(n-1) \cdots 21$ belong to the same class. There is only one class whenever $k \geq\left\lfloor\frac{n}{2}\right\rfloor$. The second term $\sum_{k=1}^{\left\lfloor\frac{n}{2}\right\rfloor-1} b_{n}^{k}$ corresponds to permutations having a non-empty $\sigma(k)$ that does not fill the semi-diagonal, i.e., $1 \leq k \leq\left\lfloor\frac{n}{2}\right\rfloor-1$; and the last term $n-1$ enumerates classes of permutations having an empty $\sigma(k)$.

Now, we will calculate $b_{n}^{k}$.

For convenience, we will say that $\gamma$ (resp. $\delta$ ) intersects the excedance board of $\pi$ if the associated block of $\gamma$ (resp. $\delta$ ) illustrated in red (resp. green) in Figure 3 has a non-empty intersection with the excedance board of $\pi$.

Using Figure 3, a simple observation provides that $\gamma$ and $\delta$ do not intersect the excedance board if and only if $\ell \leq k+1$ and $\ell+n-\ell-k \leq \ell+k+1$ which is equivalent to $n-2 k-1 \leq \ell \leq k+1$. This case can occur whenever $k+1 \geq n-2 k-1$, that is $k \geq\left\lceil\frac{n-2}{3}\right\rceil$.

We distinguish two cases.

Case 1. If $k \in\left[1,\left\lceil\frac{n-2}{3}\right\rceil-1\right]$ then there is at least one block issue from $\gamma$ or $\delta$ that intersects the excedance board of $\pi$. This means that $\gamma$ and $\delta$ are entirely determined by $\ell$ that describes the interval $[n-k-1]$. Therefore, in this case $b_{n}^{k}=n-k-1$ and this case contains exactly $\sum_{k=1}^{\left\lceil\frac{n-2}{3}\right\rceil-1}(n-k-1)$ classes.

Case 2. If $k \geq\left\lceil\frac{n-2}{3}\right\rceil$ then there exists $\ell, n-2 k-1 \leq \ell \leq k+1$, such that the two blocks associated to $\gamma$ and $\delta$ do not intersect the excedance board of $\pi$. Moreover, all permutations obtained by varying $\ell$ from $n-2 k-1$ to $k+1$ lie in a unique class for a given $k$. Now, let us assume that either $\ell \in[n-2 k-2]$ or $\ell \geq k+2$. If $\ell \in[n-2 k-2]$ then the block associated to $\gamma$ intersects the excedance board of $\pi$ that also determines $\delta$. The number 
Table 3: Number of equivalence classes for permutations avoiding two patterns in $\mathcal{S}_{3}$, where $F_{n}$ is the $n$-th Fibonacci number defined as $F_{n}=$ $F_{n-1}+F_{n-2}$ with $F_{0}=F_{1}=1$

\begin{tabular}{|c|c|c|c|}
\hline Pattern & Sequence & Sloane & $a_{n}, n \geq 1$ \\
\hline$\{123,321\}$ & & & $1,2,4,4,0,0,0, \ldots$ \\
\hline $\begin{array}{l}\{132,312\},\{213,312\}, \\
\{231,312\},\{231,321\}, \\
\{321,312\}\end{array}$ & $2^{n-1}$ & $A 000079$ & $\begin{array}{l}1,2,4,8,16,32 \\
64,128,256\end{array}$ \\
\hline $\begin{array}{l}\{123,312\},\{132,321\}, \\
\{213,321\}\end{array}$ & $(n-1) n / 2+1$ & $A 000124$ & $\begin{array}{l}1,2,4,7,11,16, \\
22,29,37\end{array}$ \\
\hline$\{132,231\},\{213,231\}$ & $F_{n}$ & $A 000045$ & $\begin{array}{l}1,2,3,5,8,13 \\
21,34,55\end{array}$ \\
\hline$\{123,231\}$ & $\left\lfloor(n-2)^{2} / 3\right\rfloor+n$ & $A 096777$ & $\begin{array}{l}1,2,3,5,8 \\
11,15,20,25\end{array}$ \\
\hline $\begin{array}{l}\{123,132\},\{123,213\}, \\
\{132,213\}\end{array}$ & $\left(5+(-1)^{n}\right) 2^{\lfloor(n-1) / 2\rfloor-1}-1$ & $A 052955$ & $\begin{array}{l}1,2,3,5,7,11 \\
15,23,31\end{array}$ \\
\hline
\end{tabular}

of classes in this subcase is exactly $n-2 k-2$ for a given $k, k \geq\left\lceil\frac{n-2}{3}\right\rceil$. If $\ell \geq k+2$ then the block associated to $\delta$ intersects the excedance board of $\pi$ that also determines $\gamma$. The number of classes in this subcase is exactly $n-k-1-(k+2)+1=n-2 k-2$. So, we have $b_{n}^{k}=2(n-2 k-2)+1$ and the total number of classes satisfying Case 2 is exactly $\sum_{k=\left\lceil\frac{n-2}{3}\right\rceil}^{\left\lfloor\frac{n}{2}\right\rfloor-1}(2(n-2 k-2)+1)$.

In accordance with these two cases, we finally obtain

$$
a_{n}=1+n-1+\sum_{k=1}^{\left\lceil\frac{n-2}{3}\right\rceil-1}(n-k-1)+\sum_{k=\left\lceil\frac{n-2}{3}\right\rceil}^{\left\lfloor\frac{n}{2}\right\rfloor-1}(2(n-2 k-2)+1) .
$$

With a simple calculation on this formula, we obtain the expected result and the sequence A096777 in [17].

\subsection{The set $S_{n}^{\sim}(132,231)-$ Fibonacci}

Theorem 4.2. The sets $S_{n}^{\sim}(132,231), n \geq 1$, are enumerated by the Fibonacci sequence (the $n$-th Fibonacci number $F_{n}$ is defined by $F_{n}=F_{n-1}+$ $F_{n-2}$ with $F_{0}=F_{1}=1$, see sequence $A 000045$ in [17]).

Proof. For $n \geq 1$, we denote by $a_{n}$ the cardinality of $S_{n}^{\sim}(132,231)$. Let $\pi$ be a permutation avoiding 132 and 231 . Thus, $\pi$ can be written either (1) $\pi=\pi_{1} \cdots \pi_{n-1} n$ or $(2) \pi=n \pi_{2} \cdots \pi_{n}$. The number of classes in $S_{n}(132,231)$ satisfying case (1) is also the number $a_{n-1}$ (we omit the last value $n$ ). Now, 
let us study the second case. There exists $j \geq 2$ such that $\pi_{j}=1$ and $\pi_{1}=n>\pi_{2}>\cdots>\pi_{j}=1<\pi_{j+1}<\cdots \pi_{n}$. It is straightforward to see that $\pi_{i} \leq i-1$ for $i \geq j$. So, we set $\pi^{\prime}=\left(\pi_{2}-1\right) \cdots\left(\pi_{j-1}-1\right)\left(\pi_{j+1}-1\right) \cdots\left(\pi_{n}-1\right)$. Obviously, $\pi^{\prime} \in S_{n-2}(132,231)$ and there is a one-to-one correspondence between classes of $S_{n}(132,231)$ having $n$ in first position, and classes of $S_{n-2}(132,231)$. Finally the number of classes in $S_{n}(132,231)$ (in this case) is equal to $a_{n-2}$ (we omit $n$ and 1 in $\pi$ ). According to the two previous cases, we have $a_{n}=a_{n-1}+a_{n-2}$ anchored with $a_{1}=1$ and $a_{2}=2$ which defines the Fibonacci sequence.

\subsection{The set $S_{n}^{\sim}(123,132)-\mathrm{A} 052955$}

Theorem 4.3. The sets $S_{n}^{\sim}(123,132), n \geq 1$, are enumerated by $2^{\frac{n+1}{2}}-1$ if $n$ odd, and by $3 \cdot 2^{\frac{n}{2}-1}-1$ otherwise (see sequence A052955 in [17]).

Proof. Let $\pi$ be a permutation avoiding 123 and 132. Let $i_{1}<i_{2}<\cdots<i_{k}$ be the indices of the right-to-left maxima of $\pi$, i.e., the indices $i_{j}, j \in[k]$, such that $\pi\left(i_{j}\right)>\pi(\ell)$ for $\ell>i_{j}$. Then $\pi$ can be obtained by gluing, from left to right, the blocks $\left(\pi\left(i_{j}\right)-1\right)\left(\pi\left(i_{j}\right)-2\right) \cdots\left(\pi\left(i_{j+1}\right)+1\right) \pi\left(i_{j}\right)$ for $1 \leq j \leq k$ by setting $\pi\left(i_{1}\right)=n$ and for convenience $i_{k+1}=n+1$ and $\pi\left(i_{k+1}\right)=0$.

We distinguish two cases: (1) $i_{1}>\left\lfloor\frac{n}{2}\right\rfloor$, and (2) $i_{1} \leq\left\lfloor\frac{n}{2}\right\rfloor$.

Case 1. We have $i_{1}>\left\lfloor\frac{n}{2}\right\rfloor$. So, $\pi$ can be written $\pi=(n-1)(n-2) \cdots$ $\left(\pi\left(i_{2}\right)+1\right) n \gamma$ where $\gamma$ has all its values less or equal than $\pi\left(i_{2}\right)$ and so that these values do not lie in the excedance board of $\pi$. We set $\pi^{\prime}=(n-1)(n-2)$ $\cdots\left(\pi\left(i_{2}\right)+1\right) n \pi\left(i_{2}\right)\left(\pi\left(i_{2}\right)-1\right) \cdots 321$. Obviously, $\pi^{\prime}$ avoids 123 and 132, and $\pi^{\prime}$ and $\pi$ belong to the same class. Varying $i_{1}$ from $\left\lfloor\frac{n}{2}\right\rfloor$ to $n$, the number of classes (in this case) is exactly $\sum_{k=\left\lfloor\frac{n}{2}\right\rfloor+1}^{n} 1=n-\left\lfloor\frac{n}{2}\right\rfloor$.

Case 2. We have $i_{1} \leq\left\lfloor\frac{n}{2}\right\rfloor$. We will prove that there is a permutation $\pi^{\prime}$ in the class of $\pi$ such that $\pi^{\prime}=(n-1)(n-2) \cdots\left(\pi\left(i_{2}\right)+1\right) n \delta i_{1}\left(i_{1}-1\right) \cdots 21$.

Let $r$ be the smallest index $j$ such that $\pi\left(i_{j}\right) \leq i_{j}$. Less formally, the point $\left(i_{r}, \pi\left(i_{r}\right)\right)$ is the highest point that does not lie in the excedance board of $\pi$.

Since $i_{1} \leq\left\lfloor\frac{n}{2}\right\rfloor$ and $\pi\left(i_{1}\right)=n$, we necessarily have $r \geq 2$. The permutation $\pi$ can be written $\pi=\gamma\left(\pi\left(i_{r}\right)-1\right) \cdots\left(\pi\left(i_{r+1}\right)+1\right) \pi\left(i_{r}\right) \sigma$ where all values in $\gamma$ appear in the excedance board of $\pi$, and all values in $\sigma$ do not lie in the excedance board of $\pi$. So, there exists an index $t=\pi_{i_{r}}, i_{r-1}<t \leq i_{r}$, such that $\pi(t)=t=\pi_{i_{r}}$.

Now let us consider the permutation $\pi^{\prime}$ obtained from $\pi$ by replacing the block $\left(\pi\left(i_{r}\right)-1\right) \cdots\left(\pi\left(i_{r+1}\right)+1\right) \pi\left(i_{r}\right)$ with the block

$$
\left(\pi\left(i_{r-1}+1\right)\right) \cdots \pi(t-1) \pi\left(i_{r}\right) \pi(t) \cdots\left(\pi\left(i_{r+1}\right)+1\right) .
$$


Moreover, we replace $\sigma$ by the decreasing sequence $\left(\pi\left(i_{r}\right)-1\right) \cdots 21$. It is straightforward to see that $\pi$ and $\pi^{\prime}$ lie in the same class. Moreover, $\pi^{\prime}$ is in the expected form $\pi^{\prime}=(n-1)(n-2) \cdots\left(\pi\left(i_{2}\right)+1\right) n \delta i_{1}\left(i_{1}-1\right) \cdots 21$ where $\delta$ is a subsequence of length $n-2 i_{1}$ that avoids 123 and 132 .

Thus, the number of classes in $S_{n}(123,132)$ such that the point $\left(i_{1}, n\right)$ lies in the excedance board is exactly the number $a_{n-2 i_{1}}$ of classes in $S_{n-2 i_{1}}(123$, 132). Varying $i_{1}$ from 1 to $\left\lfloor\frac{n}{2}\right\rfloor$, the number $b_{n}$ of classes (in this case) is given by $b_{n}=\sum_{i_{1}=1}^{\left\lfloor\frac{n}{2}\right\rfloor} a_{n-2 i_{1}}$ with $a_{1}=1$ and $a_{2}=2$.

Considering the two cases, we obtain

$$
a_{n}=n-\left\lfloor\frac{n}{2}\right\rfloor+\sum_{i_{1}=1}^{\left\lfloor\frac{n}{2}\right\rfloor} a_{n-2 i_{1}}
$$

and finally a simple calculation provides $a_{n}=2 a_{n-2}+1$ with $a_{1}=1$ and $a_{2}=2$ which is the sequence A052955 in [17].

\section{Acknowledgement}

We would like to thank the anonymous referees for their very careful reading of this paper and their helpful comments and suggestions.

\section{References}

[1] C. Banderier, M. Bousquet-Mélou, A. Denise, P. Flajolet, D. Gardy, and D. Gouyou-Beauchamps. Generating functions of generating trees. Discrete Mathematics, 246(1-3), 29-55, 2002. MR1884885

[2] E. Barcucci, A. Bernini, and M. Poneti. From Fibonacci to Catalan permutations. Pure Mathematics and Applications, 17, 1-17, 2006. MR2380345

[3] E. Barcucci, A. Del Lungo, E. Pergola, and R. Pinzani. From Motzkin to Catalan permutations. Discrete Mathematics, 217, 33-49, 2000. MR1766258

[4] N. Bergeron and M. Zabrocki. The Hopf algebras of symmetric functions and quasisymmetric functions in non-commutative variables are free and cofree. J. Algebra Appl., 08(4), 581-600, 2009. MR2555523

[5] R. Ehrenborg and E. Steingrimsson. The excedance set of a permutation. Advances in Applied Mathematics, 24, 284-299, 2000. MR1751147 
[6] S. Elizalde. Statistics on pattern-avoiding permutations. Ph.D. thesis, MIT, 2004, https://www.math.dartmouth.edu/ $\sim_{\text {sergi/papers/thesis. }}$ pdf. MR2717168

[7] S. Elizalde and E. Deutsch. A simple and unusual bijection for Dyck paths and its consequences. Ann. Comb., 7(3), 281-297, 2003. MR2028229

[8] S. Kitaev. Patterns in Permutations and Words. Springer-Verlag, 2011. MR3012380

[9] D. E. Knuth. The Art of Computer Programming. Vol. 1, AddisonWesley, Reading MA, 1973, Third edition, 1997. MR3077152

[10] T. Mansour. Combinatorics of Set Partitions. Discrete Mathematics and Its Applications, Chapman \& Hall/CRC, 2013. MR2953184

[11] T. Mansour. Permutations avoiding a pattern from $S_{k}$ and at least two patterns from $S_{3}$. Ars. Combin., 62, 227-239, 2002. MR1881962

[12] T. Mansour and A. Vainshtein. Restricted 132-avoiding permutations. Adv. Appl. Math., 26, 258-269, 2001. MR1818747

[13] R. Simion and F. W. Schmidt. Restricted permutations. European Journal of Combinatorics, 6, 383-406, 1985. MR0829358

[14] J. West. Permutations with forbidden subsequences and stack-sortable permutations. Ph.D. thesis, MIT, 1990. MR2716312

[15] J. West. Generating trees and forbidden subsequences. Proceeding of Sixth FPSAC, 441-450, 1994.

[16] J. West. Generating trees and the Catalan and Schröder numbers. Discrete Math., 146, 247-262, 1995. MR1360119

[17] N. J. A. Sloane. On-Line Encyclopedia of Integer Sequences. Published electronically at http://oeis.org/.

[18] R. Stanley. Enumerative Combinatorics. Cambridge University Press, Vol. 1, 1997. MR1442260

JEAN-LUC BARIL

LE2I UMR-CNRS 6306

UNIVERSITÉ DE BOURGOGNE

B.P. 47 870, 21078 DiJON

FRANCE

E-mail address: barj1@u-bourgogne.fr 
TOUFIK MANSOUR

Department of Mathematics

UNIVERSITY OF HAIFA

3498838 HAIFA

ISRAEL

E-mail address: tmansour@univ.haifa.ac.il

Armen Petrossian

LE2I UMR-CNRS 6306

Université DE Bourgogne

B.P. 47 870, 21078 DiJON

FRANCE

E-mail address: armen.petrossian@u-bourgogne.fr

Received 10 DeCember 2013 